ISSN 0103-5150

Fisioter. Mov., Curitiba, v. 25, n. 2, p. 301-309, abr./jun. 2012 Licenciado sob uma Licença Creative Commons

\title{
Análise da confiabilidade do teste clínico de queda do navicular
}

\author{
Reliability analysis of the clinical application of the navicular drop test
}

\author{
George Schayer Sabino ${ }^{[a]}$, Ivan Casas Rocha ${ }^{[b]}$, Cristiano Queiroz Guimarães ${ }^{[c]}$, \\ Marcus Alessandro de Alcântara ${ }^{[\mathrm{d}]}$, Diogo Carvalho Felício ${ }^{[\mathrm{e}]}$
}

[a] Fisioterapeuta da Propulsão, Mestre em Ciências da Reabilitação pela Universidade Federal de Minas Gerais (UFMG), docente do Centro Universitário Newton Paiva e da Faculdade Pitágoras, Belo Horizonte, MG - Brasil, e-mail: george@propulsao.com

[b] Fisioterapeuta da Propulsão, Belo Horizonte, MG - Brasil, e-mail: ivan@propulsao.com

[c] Fisioterapeuta da Propulsão, Mestre em Ciências da Reabilitação pela Universidade Federal de Minas Gerais (UFMG), docente da Faculdade Itabirana de Saúde, Belo Horizonte, MG - Brasil, e-mail: cristiano@propulsao.com

[d] Mestre em Ciências da Reabilitação pela Universidade Federal de Minas Gerais (UFMG), docente da Universidade Federal do Vale do Jequitinhonha e Mucuri (UFVJM), Teófilo Otoni, MG - Brasil, e-mail: alcantaramarcus@hotmail.com

[e] Fisioterapeuta da Prefeitura Municipal de Betim, Mestrando em Ciências da Reabilitação pela Universidade Federal de Minas Gerais (UFMG), docente da Faculdade Pitágoras, Betim, MG - Brasil, e-mail: diogofelicio@yahoo.com.br

\section{Resumo}

Introdução: A utilização de medidas confiáveis e práticas são necessárias para a tomada de decisões clínicas. O Teste da Queda do Navicular (TQN) fornece informações importantes na avaliação dos membros inferiores. Assim, os objetivos do presente estudo foram verificar a confiabilidade do TQN, conforme realizado na clínica, e analisar uma forma de minimizar a interferência do avaliador. Materiais e métodos: Participaram do estudo 15 indivíduos (30 pés), selecionados por conveniência. O TQN foi aplicado de forma independente por dois examinadores experientes na realização do teste, o qual foi realizado apenas uma vez em cada pé, utilizando-se um paquímetro de plástico. Foi realizada uma análise descritiva dos dados. Para avaliação da confiabilidade interexaminadores, foi utilizado o índice de correlação intraclasse (ICC 2,1 ) e a discrepância das medidas entre avaliadores foi analisada pelo erro típico da medição. Resultados: A média e o desvio padrão para o primeiro e segundo examinador foram 8,7 \pm 5,3 e 9,7 \pm 4,7 milímetros. 0 teste apresentou excelente confiabilidade interexaminador (ICC = 0,93). 0 erro típico da medição foi 2,16 milímetros. 
Conclusão: Os procedimentos para minimizar a interferência do avaliador resultaram, contraditoriamente, em uma piora dos índices de confiabilidade. 0 TQN pode ser simplificado por meio do uso de instrumentos comuns na clínica e registrado a partir de uma única medida. Mesmo apresentando boa confiabilidade, o TQN apresenta um erro típico da medida, que deve ser levado em consideração para a tomada de decisão. Mudanças no teste, por vezes necessárias em um ambiente clínico, deverão ser analisadas quanto à conservação de suas propriedades.

Palavras-chave: Reprodutibilidade dos testes. Exame físico. Pé.

\section{Abstract}

Introduction: Reliable and practical measures are necessary for making clinical decisions. The Navicular Drop Test (NDT) provides important information during evaluation of lower limbs. The purpose of the present article was to assess the psychometric properties of NDT as usually done in clinical environment, and to analyze a way to minimize the evaluator interference. Materials and methods: Fifteen people (30 feet), selected by convenience, participated. The NDT was performed independently by two investigators experienced in the test realization. The test was performed once in each foot, using a plastic caliper. Descriptive analysis of data was done. In order to evaluate the inter-examiner reliability, intraclass correlation index (ICC $\left.{ }_{2,1}\right)$ was used. The divergence between the measures was examined by the typical error measurement. Results: The mean and standard deviation for the first and second examiner was $8.7 \pm 5.3$ and $9.7 \pm 4.7 \mathrm{~mm}$. The test showed excellent inter-examiner reliability (ICC $=0.93)$. The typical error of measurement was $2.16 \mathrm{~mm}$. Conclusion: The procedures to minimize the interference of evaluator, paradoxically, showed worse indices of reliability. The NDT can be simplified by using common clinical tools and recorded from a single measure. Even though it showed a good reliability, the NDT contains a typical error of measurement which has to be considered for decision making. Changes in the test, sometimes required in a dynamic clinical environment, should be analyzed for maintenance of test properties.

Keywords: Reproducibility of results. Physical examination. Foot.

\section{Introdução}

0 atendimento de um paciente por profissionais de saúde envolve a realização de uma avaliação para identificar prejuízos sobre a funcionalidade, onde são executados testes clínicos (1).

Para que um teste possa ser utilizado clinicamente, ele deve ser válido e confiável. Validade é a capacidade de um teste medir aquilo que ele se propõe a medir (2). Confiabilidade, por sua vez, refere-se à capacidade de reproduzir os mesmos escores em repetidas administrações de um teste que avalia uma variável que se mantém constante (3). A confiabilidade é um pré-requisito para a validade (2).

A confiabilidade é fundamental para a prática clínica (2). Um teste que apresente valores distintos em mensurações subsequentes não permitirá determinar se o indivíduo avaliado apresenta um valor anormal. Uma medida pouco confiável poderá levar, também, a falsas conclusões a respeito dos resultados de uma intervenção. Um indivíduo pode, por exemplo, frustrar-se com resultados aparentemente negativos de uma intervenção que, de fato, foi efetiva, ou acreditar na evolução de um tratamento de uma condição que está estagnada (1). Segundo Lunes et al. (4), a escassez de testes confiáveis pode explicar por que existem poucos resultados de estudos que conseguem associar alterações posturais a lesões ou disfunções musculoesqueléticas específicas.

Um teste válido e confiável que é facilmente realizado na clínica fisioterápica é o teste da queda do navicular (TQN) (5). Além de apresentar propriedades de medidas adequadas, são disponibilizados na literatura os dados normativos para o TQN (6, 7-10).

0 TQN mede o deslocamento vertical da tuberosidade do navicular durante a descarga de peso e pode ser utilizado para avaliar a quantidade de pronação da articulação subtalar $(9,11)$. Dessa forma, a utilização desse teste contribui para a análise do movimento dos pés e para o entendimento de algumas disfunções 
associadas a essa dinâmica. Existem estudos que sugerem o uso do TQN para a triagem de atletas com risco de lesão do ligamento cruzado anterior $(7,12$, 13). Considerando que, em termos biomecânicos, o corpo humano é um composto multissegmentar que apresenta interações não apenas entre regiões adjacentes, mas também entre partes distantes, e que essas inter-relações podem exercer influência nos sintomas musculoesqueléticos dos indivíduos (14), o TQN pode ser empregado, inclusive, na avaliação e interpretação de disfunções distais aos pés (15).

Para uma medida ser difundida, entretanto, além de válida, confiável e relevante, ela deve ser também prática e rápida. Testes precisos e fáceis de serem empregados tornam mais ágeis os procedimentos de avaliação e o monitoramento dos efeitos da intervenção, contribuindo, assim, para sua consolidação na prática $(1,3)$.

O TQN é um teste prático, contudo, conforme descrito na literatura, deve ser obtido por meio da média de três medidas (6). Outra exigência é que o avaliador posicione a articulação subtalar do paciente na posição neutra (11). 0 método de posicionamento da subtalar em neutro pela palpação do tálus foi desenvolvido para agilizar o processo de avaliação, entretanto, esse método requer localização de estruturas ósseas pelo tato, o que envolve componentes subjetivos de avaliação. Estudos propõem que, quanto menor o número de procedimentos, maior a facilidade de emprego de um teste e que, quanto menor a interferência do examinador, maior a precisão da medida (16).

É atual a discussão sobre melhorias na utilização do TQN (5). A avaliação e o aprimoramento dos procedimentos utilizados na prática clínica fazem parte do método científico e são importantes para a evolução dos cuidados em saúde. Assim, o objetivo do presente estudo foi verificar se a confiabilidade do TQN descrita na literatura é reproduzida conforme normalmente realizado na clínica, e tentar desenvolver uma forma de minimizar a interferência do avaliador durante a realização do teste.

\section{Materiais e métodos}

\section{Teste da Queda do Navicular (TQN)}

Com o participante em posição sentada com os pés apoiados no chão, a região palpável mais pro- eminente da tuberosidade do navicular foi marcada com um pequeno ponto utilizando uma caneta esferográfica azul. A articulação subtalar foi, então, colocada em neutro pelo método de palpação do tálus (11) (Figura 1A) e a distância vertical da marcação da tuberosidade do navicular ao solo foi mensurada. A posição neutra da articulação subtalar foi considerada quando o avaliador, ao inverter e everter o pé do participante, palpava o tálus igualmente medial e lateralmente (11).

Após a primeira mensuração, o indivíduo foi instruído a ficar de pé, em duplo apoio, em uma superfície firme, permitindo ao pé sair da posição neutra (Figura 1B). A altura da tuberosidade do navicular em relação ao solo foi, então, novamente medida. A diferença entre as duas medidas foi registrada em milímetros $(10,12,17)$.

\section{Teste da Queda do Navicular Adaptado (TQNA)}

Foi utilizada a tuberosidade do navicular como referência para o movimento do pé, mas, ao invés de posicionar a articulação subtalar em neutro na postura sentada, o pé foi mantido em uma posição relaxada, sem descarga de peso e sem a interferência
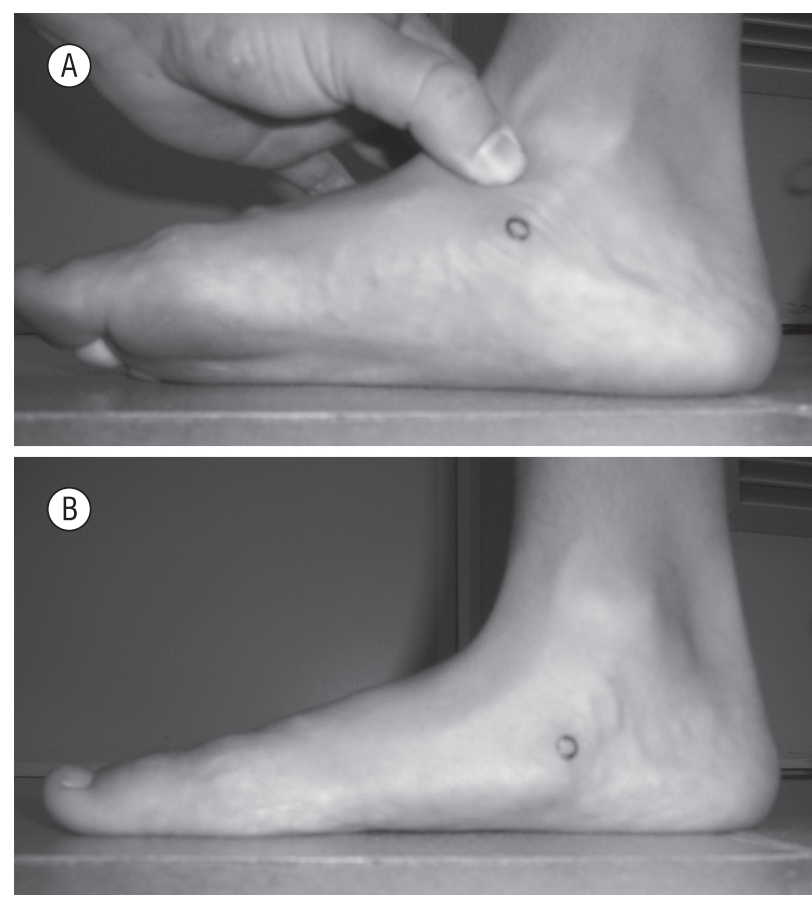

Figura 1 - Fotos ilustrativas do teste da queda do navicular. A) Subtalar em neutro. B) Altura da marcação durante o duplo apoio 
do avaliador; ou seja, a diferença fundamental entre o TQN e o TQNA está na supressão do posicionamento da subtalar em neutro. Foi, então, mensurada a distância da tuberosidade do navicular ao solo.

A diferença entre as medidas na posição assentada, sem interferência do examinador, e em ortostatismo com descarga de peso foi calculada e registrada em milímetros, sendo denominado Teste da Queda do Navicular Adaptado.

\section{Avaliadores}

Dois avaliadores, graduados em Fisioterapia, com mais de quatro anos de experiência em análise de marcha e aplicação do TQN, realizaram os testes de forma independente em todos os participantes do estudo. A fim de padronizar a realização dos testes, os dois avaliadores foram orientados a ler e a seguir as orientações apresentadas anteriormente. Entretanto, não foi realizado um treinamento conjunto para a padronização de suas medidas. Para evitar o contato entre os examinadores, os procedimentos foram realizados em salas distintas.

\section{Amostra}

A seleção da amostra foi realizada por conveniência. Participaram deste estudo 15 voluntários (30 pés), maiores de 18 anos, de ambos os sexos, recrutados pelos pesquisadores em clínicas e universidades de fisioterapia da cidade de Belo Horizonte/MG. Os indivíduos com história de lesão ou dor nos pés foram excluídos. Todos os voluntários assinaram um termo de consentimento livre e esclarecido concordando em participar do estudo.

\section{Procedimentos}

Cada medida foi realizada apenas uma vez pelos examinadores. Havia um intervalo para apagar a marcação da tuberosidade do navicular antes da realização das medidas pelo próximo examinador. A ordem de realização do TQN e do TQNA, bem como a ordem dos avaliadores foi determinada de forma aleatória (sorteio) para cada sujeito. Um avaliador não teve contato com as medidas realizadas pelo outro avaliador até o término de todos os procedimentos.

\section{Instrumentos}

Para a avaliação da altura da tuberosidade do navicular ao solo, foi utilizado um paquímetro de plástico (da marca Vonder), mantido perpendicular ao solo, com seu braço fixo encostado na superfície de apoio. 0 braço móvel do paquímetro foi elevado até a marcação da tuberosidade do navicular e foi registrado o valor determinado pela abertura dos braços do instrumento.

\section{Análise dos dados}

Foi realizada a análise descritiva da tendência central dos dados e da variância da amostra por meio da média e do desvio padrão das medidas do TQN e do TQNA para a população estudada e das medidas separadas de cada examinador. Para a análise da amplitude de valores das medidas, foram registrados os valores máximo e mínimo obtidos para as medidas do TQN e do TQNA.

Os erros sistemáticos das medidas entre examinadores foram avaliados por métodos visuais de análise em gráficos de linha plotados no Excel 2000 (3), confirmados pela análise de variância para medidas repetidas (ANOVA) realizada no SPSS 15.0. Foram consideradas diferenças significativas quando o $\mathrm{p}<$ 0,05. A ausência de erros sistemáticos de medida foi estabelecida quando verificada ausência de variações consistentes entre as medidas dos examinadores nos gráficos de linha associada à ausência de diferenças significativas entre as medidas dos examinadores (3).

Finalmente, para a avaliação da confiabilidade, foi analisado o índice de correlação intraclasse (Two-way mixed, absolute agreement, ICC $_{2,1}$ ) e seu intervalo de confiança (18). Foi considerada boa confiabilidade para o teste ICC maiores que 0,8 (2). A discrepância das medidas entre avaliadores, denominada erros aleatórios, foi avaliada por meio do erro típico (erro padrão da medição) e pelo coeficiente de variação, o qual consistia do erro típico, dividido pela média da população.

\section{Resultados}

A média ( $\underline{x}$ ) e o desvio padrão da média (dp) para as medidas do TQN e do TQNA para a população em 
estudo, bem como a x e o dp para as medidas do primeiro e segundo avaliador encontram-se registrados na Tabela 1.

Os valores do TQN variaram de $-3,5$ a $19 \mathrm{~mm}$ e os valores do TQNA, de 0 a 14,5 mm.

Não foram observados padrões de comportamento para as diferenças entre as medidas dos examinadores na análise visual dos gráficos de linha (Gráfico 1) ou diferenças significativas entre as medidas dos dois avaliadores ( $\mathrm{p}>0,05)$, indicando a não existência de erros sistemáticos de medidas interexaminadores.

O ICC $_{2,1}$ e seu Intervalo de Confiança (IC) para as medidas realizadas encontram-se dispostas na Tabela 2, juntamente com o ICC $_{2,1}$ e IC do TQN e do TQNA.

0 erro típico entre as medidas dos examinadores foi de 2,16 $\mathrm{mm}$ para o TQN e de 2,8 $\mathrm{mm}$ para o

Tabela 1 - Média e desvio padrão dos dados referentes ao teste da queda do navicular (TQN) e ao teste da queda do navicular adaptada (TQNA) (em mm)

\begin{tabular}{lcc}
\hline & TQN & TQNA \\
\hline População & $9,0(4,9)$ & $7,4(2,6)$ \\
Avaliador 1 & $8,7(5,3)$ & $7,2(3,1)$ \\
Avaliador 2 & $9,7(4,7)$ & $7,5(3,0)$ \\
\hline
\end{tabular}

Fonte: Dados da pesquisa.

Tabela 2 - Índice de Correlação Intraclasse (ICC 2,1$)$ e Intervalo de Confiança (IC)

\begin{tabular}{lcc}
\hline & ICC $_{2,1}$ & IC \\
\hline Medida I (neutro) & 0,924 & $0,841-0,963$ \\
Medida 2 (em posição sentada) & 0,953 & $0,892-0,979$ \\
Medida 3 (em posição ortostática) & 0,928 & $0,824-0,968$ \\
TQN & 0,931 & $0,848-0,968$ \\
TQNA & 0,541 & $0,041-0,781$ \\
\hline
\end{tabular}

Fonte: Dados da pesquisa.

Legenda: Medida 1 = medidas da altura da tuberosidade do navicular ao solo com a subtalar em neutro; Medida 2 = medida da altura da tuberosidade do navicular ao solo com o pé relaxado sem descarga de peso; Medida $3=$ medida da altura da tuberosidade do navicular ao solo em ortostatismo com o pé relaxado e com descarga de peso; TQN = Teste da Queda do Navicular; TQNA = Teste da Queda do Navicular Adaptado.
TQNA. 0 coeficiente de variação entre as medidas dos examinadores foi de 0,24 para o TQN e de 0,38 para o TQNA.

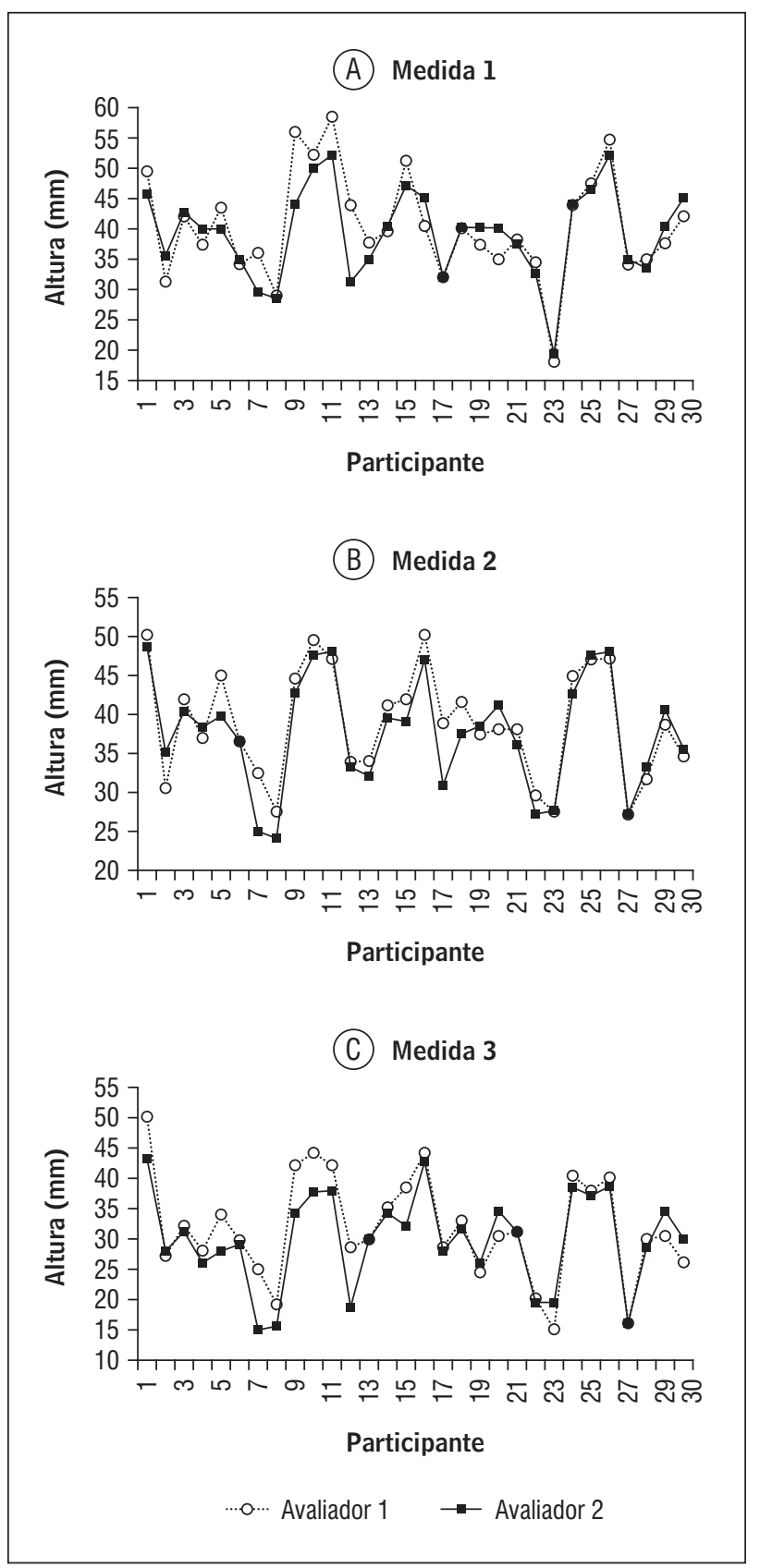

Gráfico 1 - Medidas da altura da tuberosidade do navicular ao solo dos avaliadores 1 e 2. A) Medida $1=$ altura da tuberosidade do navicular ao solo com a subtalar em neutro; B) Medida 2 = altura da tuberosidade do navicular ao solo com o pé relaxado, com o indivíduo em posição sentada; C) Medida 3 = altura da tuberosidade do navicular ao solo com o indivíduo em pé

Fonte: Dados da pesquisa. 


\section{Discussão}

O presente estudo buscou verificar se a confiabilidade de um teste, reportada pela literatura científica (10), seria alcançada por dois avaliadores experientes, realizando-o conforme é executado em suas rotinas, e se um novo teste modificado apresentaria medidas apropriadas para ser empregado clinicamente.

Os valores médios do TQN para a amostra estudada, considerando as medidas realizadas pelos dois examinadores, foram de 9,0 $\pm 4,9 \mathrm{~mm}$. Esses valores são condizentes com os encontrados em estudos normativos do TQN, que variam de 6 a $9 \mathrm{~mm}$, com desvio padrão de 3 a 4 mm (5, 7-10).

Não foram observados erros sistemáticos para as medidas realizadas. No TQN da forma proposta, o erro sistemático poderia ter advindo do posicionamento e da leitura do instrumento de medida. Allen et al. (7) utilizaram um outro tipo de instrumento, mais complexo, para padronizar a medida do TQN. Entretanto, o paquímetro de plástico, um utensílio simples e de baixo custo, não se mostrou uma fonte de erros sistemáticos.

A realização de apenas uma medida é costumeira na prática clínica (3). Sua vantagem é aumentar a praticidade do teste ao agilizar a mensuração. Essa forma de proceder não comprometeu a confiabilidade do TQN, pois mesmo realizando apenas uma medida, os índices de confiabilidade foram adequados. Assim, pode-se dizer que os procedimentos para o TQN mostraram-se apropriados para o emprego clínico, mesmo quando simplificados na forma do estudo atual.

Todavia, todas as medidas estão sujeitas a erros (3). Foi observado um erro típico da medição, o que significa que a medida do TQN de dois avaliadores, para um mesmo participante do estudo, divergiu em aproximadamente $2,1 \mathrm{~mm}$. 0 coeficiente de variação para o TQN foi de $24 \%$, o que indica que a medida interexaminadores apresentará uma dispersão considerável. Vários pontos podem ter influenciado essa variação entre as medidas dos examinadores, como a localização e a marcação da tuberosidade do navicular (19).

A divergência de medidas interavaliadores não invalida o teste, mas deve ser considerada durante a seleção de uma intervenção. Por exemplo, o TQN pode ser utilizado para a triagem de atletas com risco de apresentar algumas lesões $(12,13)$. Conforme discutido, os valores médios e o desvio padrão da medida do TQN para uma população saudável são de 7,3 \pm 3 ,8 mm (5), ou seja, uma queda do navicular de até $11,1 \mathrm{~mm}$ pode ser considerada adequada dentro de uma distribuição normal, ponderando um desvio padrão. Valores maiores que esse tendem a serem considerados como fatores de risco para algumas lesões. Entretanto, como um avaliador pode divergir em sua medida em 2,1 mm (erro típico da medida), acrescenta-se a esse valor extremo de 11,1 $\mathrm{mm}$, mais 2,1 $\mathrm{mm}$, de forma que um atleta com um escore de 13,2 mm no TQN, em uma triagem clínica ou esportiva, poderá, ainda, ser normal.

Assim, sugere-se que a medida isolada de um teste não seja utilizada para a tomada de decisão clínica. Para minimizar a influência do erro típico da medição, pode-se associar os resultados de diferentes testes feitos durante a avaliação clínica para a tomada de decisão. Em outras palavras, um atleta que apresente uma medida no TQN de 13,2 mm deverá ter esse resultado analisado conjuntamente com sua história pregressa, sintomatologia, nível de atividade física (atual e futuro) e com a análise visual qualitativa das características de sua marcha, a fim de se determinar o risco de ele apresentar alguma lesão.

Brody (11), em um dos primeiros estudos realizados para se avaliar a queda do navicular, propôs que uma diferença maior que $15 \mathrm{~mm}$ deveria ser considerada como uma pronação excessiva. Todavia, ele não fornecia dados para suportar essa afirmação, a qual foi, por consequência, questionada em trabalhos futuros $(5,9)$. Uma queda do navicular de 13,2 $\mathrm{mm}$, como a exemplificada anteriormente, é aceitável dentro das premissas iniciais de Brody (11) e pode ser ou não qualificada como aceitável, dependendo da situação clínica específica, considerando os resultados encontrados no presente estudo.

Foi observado um alto índice de confiabilidade interexaminador para todas as medidas da altura do navicular analisadas de forma isolada, com o ICC variando de 0,924 a 0,953. Sell et al. (10) encontraram resultados semelhantes para as medidas interexaminadores da altura do navicular em repouso $\operatorname{ICC}_{(2,1)}=$ $0,95)$ e com a subtalar em neutro $\left(\operatorname{ICC}_{(2,1)}=0,92\right)$. Os altos índices de confiabilidade dessas medidas demonstram que os procedimentos de marcação e mensuração da altura da tuberosidade do navicular ao solo, bem como do posicionamento da subtalar em neutro são consistentes.

O ICC interexaminador para o TQN no presente estudo foi de 0,931 . Testes com confiabilidade maior 
que 0,8 podem ser considerados de boa confiabilidade $(2,6)$. Entretanto, nem todos os estudos disponíveis na literatura apontam resultados similares aos encontrados. A confiabilidade intraexaminador do TQN no estudo de Shultz et al. (20) foi maior que 0,9 , porém, a confiabilidade interexaminador observada em seu estudo pode ser considerada baixa (entre 0,56 e 0,76 ).

A precisão dos resultados interexaminadores no presente trabalho, a qual diverge do estudo previamente citado (20), é um achado interessante considerando que os procedimentos propostos para o experimento atual almejavam simular um ambiente clínico. Os índices de confiabilidade maiores que os descritos por Shultz et al. (20) podem ser decorrentes da experiência na realização dos procedimentos por parte dos avaliadores (o estudo anterior, bem como outros relacionados ao assunto, utilizavam avaliadores inexperientes) $(9,10)$. Quando os resultados de confiabilidade intraexaminadores diferem dos resultados interexaminadores, pode-se concluir que mais treinamento (ou experiência) é necessário para melhorar a qualidade das medidas. Medidas de confiabilidade interavaliadores apresentam melhores resultados quando realizadas por indivíduos com mais tempo de experiência (20). A diferença de resultados entre avaliadores com e sem experiência no caso do TQN pode ser creditada à colocação da subtalar em neutro pelo método da palpação e à marcação da tuberosidade do navicular $(5,16,17)$.

Outro fator, estatístico, pode ter contribuído para a diferença de resultados entre estudos (18). Atualmente, não existe um consenso na literatura a respeito da melhor medida para a avaliação da confiabilidade (3). O ICC, apesar de difundido e de permitir a comparação da confiabilidade entre diferentes instrumentos, se mostra incompleto e sujeito a variâncias da medida em amostras distintas. Um mesmo instrumento utilizado em duas populações apresentará ICC diferentes dependendo da variância de cada amostra (21). A confiabilidade de medidas dicotômicas ou ordinais é verificada por meio da coincidência ou não dos resultados entre as mensurações. Em medidas contínuas, os valores de medidas subsequentes não irão coincidir exatamente, apenas se aproximarão. A confiabilidade de um teste contínuo será baseada em quanto às medidas repetidas se aproximam e ainda podem ser consideradas equivalentes. Todavia, o quanto de dispersão entre essas medidas é aceitável não é estabelecido por critérios clínicos, mas pela variabilidade da população estudada. Em uma população heterogênea, uma variação maior entre os resultados é esperada, e, dessa maneira, mesmo resultados discrepantes poderão ser considerados consistentes. Dessa forma, não é adequado relatar a confiabilidade de uma medida contínua simplesmente pelo ICC, pois, dependendo da amostra, essa porcentagem poderá ser superestimada.

Como forma de complementar o ICC, é importante que estudos de confiabilidade avaliem a dispersão dos valores das medidas repetidas na mesma unidade do instrumento, isto é, no caso do TQN, a variância em milímetros. Análises relacionadas à confiabilidade que empreguem medidas na mesma unidade de medida do instrumento são importantes para determinar se, durante o tratamento, ocorreu uma mudança clínica real em um paciente (uma mudança superior ao erro típico da medida que foi repetida) (21).

Dessa forma, o presente estudo empregou diversos testes para a análise da confiabilidade. Todas as análises de confiabilidade foram realizadas para o TQN e para o TQNA para que essas duas medidas pudessem ser comparadas quanto às suas propriedades. Surpreendentemente, o TQNA apresentou valores piores em termos de confiabilidade, em relação ao TQN.

A partir desses achados, torna-se necessário salientar a importância da análise metodológica da modificação de um procedimento de avaliação (ou, por extrapolação, de uma intervenção), para que este possa ser utilizado de forma apropriada. É comum a adaptação de procedimentos descritos na literatura de acordo com a realidade de trabalho em diversas clínicas de Fisioterapia do Brasil. Resolver situações problemáticas de forma criativa para agilizar e viabilizar a prática deve, sim, ser considerado um mérito para o seu autor; contudo, a resolução proposta deve ser testada quanto à sua efetividade, respeitando parâmetros metodológicos criteriosamente estipulados. 0 ato de realizar uma testagem rigorosa da mudança efetuada em um procedimento tenderá a aperfeiçoar a prática e torná-la mais correta no futuro.

Em 2008, McPoil et al. (5) publicaram um artigo com o objetivo de aperfeiçoar o TQN e validar essa nova medida. Foi sugerido um método de avaliação que retirava a subjetividade dos procedimentos de palpação da articulação subtalar e a imprecisão da marcação da tuberosidade do navicular por meio do registro digital da imagem do pé, considerando como referência para o movimento do pé a diminuição da altura do dorso do pé quando o indivíduo passava da 
postura sentada para a de pé. Esse método assemelha-se, em parte, ao proposto no presente estudo, com o incremento da fotografia digital. 0 uso de imagens digitalizadas tende a ser cada dia mais comum na prática clínica e apresenta uma série de benefícios, como a possibilidade de se demonstrar visualmente os achados da avaliação ao paciente. Todavia, a necessidade do aparato de uma câmera tem a desvantagem de aumentar o tempo gasto nos procedimentos de avaliação para se transferir a imagem para um computador e analisá-la em um programa gráfico específico, ao passo que o teste clínico sem o uso da fotografia possibilita a verificação dos resultados na hora, fato importante em uma prática que normalmente demanda procedimentos ágeis. A possibilidade de se realizar um teste clínico com ou sem uma câmera fotográfica aumenta a gama de possibilidades para o fisioterapeuta e pode ser apropriado em um ambiente clínico heterogêneo e dinâmico.

\section{Conclusão}

O TQN pode ser simplificado por meio do uso de instrumentos comuns na clínica - como um paquímetro de plástico - e registrado a partir de uma única medida, mesmo sem o treinamento padronizado de seu executor, desde que este tenha experiência clínica. Mesmo apresentando boa confiabilidade, o TQN apresenta um erro típico da medida e um coeficiente de variação apreciável, que devem ser levados em consideração para a tomada de decisão. A retirada de um procedimento comum no teste, relacionado ao posicionamento da articulação subtalar em neutro, não se mostrou apropriada; assim, esse procedimento deve ser mantido para que os valores do teste sejam confiáveis. Os profissionais devem ter cautela ao alterar um procedimento descrito na literatura científica, pois essa ação pode descaracterizá-lo e alterar suas propriedades.

\section{Referências}

1. Sampaio RF, Mancini MC, Fonseca S. Produção científica e atuação profissional: aspectos que limitam esta integração na fisioterapia e na terapia ocupacional. Rev Bras Fisioter. 2005;6(3):113-8.
2. Portney LG, Watkins MP. Foundations of clinical research: application to practice. New Jersey: PrenticeHall; 2000.

3. Batterhama AM, George KP. Reliability in evidencebased clinical practice: a primer for allied health professionals. Phys Ther Sport. 2003;4:122-8.

4. Iunes DH, Castro FA, Salgado HS, Moura IC, Oliveira AS, Bevilaqua-Grossi D. Confiabilidade intra e interexaminadores e repetibilidade da avaliação postural pela fotogrametria. Rev Bras Fisioter. 2005;9(3):327-34.

5. McPoil TG, Cornwall MW, Medoff L, Vicenzino B, Forsberg K, Hilz D. Arch height change during sit-tostand: an alternative for the navicular drop test. J Foot Ankle Res. 2008;1(1):3.

6. Shrader JA, Popovich JM Jr, Gracey GC, Danoff JV. Navicular drop measurement in people with rheumatoid arthritis: interrater and intrarater reliability. Phys Ther. 2005;85(7):656-64.

7. Allen MK, Glasoe WM. Metrecom measurement of navicular drop in subjects with anterior cruciate ligament injury. J Athl Train. 2000;35(4):403-6.

8. Mueller MJ, Host JV, Norton BJ. Navicular drop as a composite measure of excessive pronation. J Am Podiatr Med Assoc. 1993;83(4):198-202.

9. Picciano AM, Rowlands MS, Worrell T. Reliability of open and closed kinetic chain subtalar joint neutral positions and navicular drop test. J Orthop Sports Phys Ther. 1993;18(4):553-8.

10. Sell KE, Verity TM, Worrell TW, Pease BJ, Wigglesworth J. Two measurement techniques for assessing subtalar joint position: a reliability study. J Orthop Sports Phys Ther. 1994;19(3):162-7.

11. Brody DM. Techniques in the evaluation and treatment of the injured runner. Orthop Clin North Am. 1982;13(3):541-58.

12. Beckett ME, Massie DL, Bowers KD, Stoll DA. Incidence of hyperpronation in the ACL injured knee: a clinical perspective. J Athl Train. 1992;27(1):58-62.

13. Smith J, Szczerba JE, Arnold BL, Perrin DH, Martin DE. Role of hyperpronation as a possible risk factor for anterior cruciate ligament injuries. J Athl Train. 1997;32(1):25-8. 
14. Khamis S, Yizhar Z. Effect of feet hyperpronation on pelvic alignment in a standing position. Gait Posture. 2007;25(1):127-34.

15. Sabino GS, Guimarães CQ, Bicalho LI, Rocha IC, Silva ALSR. Relação entre o alinhamento pélvico e o comprimento ósseo dos membros inferiores de um atleta de futebol: estudo de caso. Fisioter Brasil. 2007;8:37-8.

16. Smith-Oricchio K, Harris BA. Interrater reliability of subtalar neutral, calcaneal inversion and eversion. J Orthop Sports Phys Ther. 1990;12(1):10-5.

17. Elveru RA, Rothstein JM, Lamb RL. Goniometric reliability in a clinical setting. Subtalar and ankle joint measurements. Phys Ther. 1988;68(5):672-7.

18. Stratford PW, Goldsmith CH. Use of the standard error as a reliability index of interest: an applied example using elbow flexor strength data. Phys Ther. 1997;77(7):745-50.

19. O'Haire C, Gibbons P. Inter-examiner and intra-examiner agreement for assessing sacroiliac anatomical landmarks using palpation and observation: pilot study. Man Ther. 2000;5(1):13-20.
20. Shultz SJ, Nguyen AD, Windley TC, Kulas AS, Botic TL, Beynnon BD. Intratester and intertester reliability of clinical measures of lower extremity anatomic characteristics: implications for multicenter studies. Clin J Sport Med. 2006;16(2):155-61.

21. Eliasziw M, Young SL, Woodbury MG, Fryday-Field K. Statistical methodology for the concurrent assessment of interrater and intrarater reliability: using goniometric measurements as an example. Phys Ther. 1994;74(8):777-88.

Recebido: 15/03/2011 Received: 03/15/2011

Aprovado: 04/08/2011

Approved: 08/04/2011 